\title{
Physiological and anatomical investigation of the auditory brainstem in the Fat-tailed dunnart (Sminthopsis crassicaudata)
}

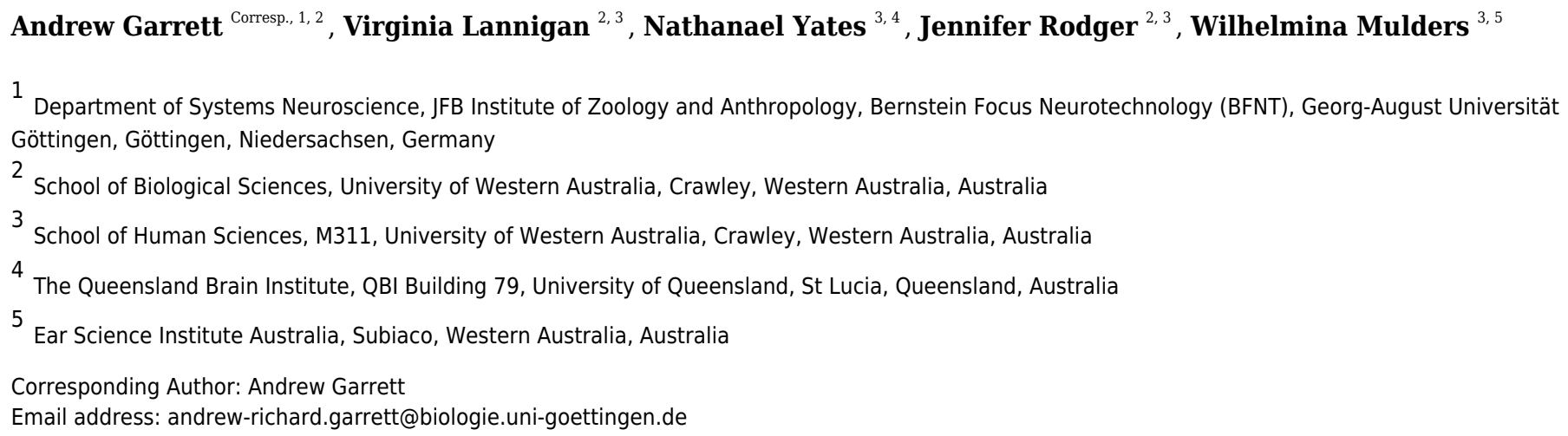

The fat-tailed dunnart (Sminthopsis crassicaudata) is a small (10-20g) native marsupial endemic to the south west of Western Australia. Currently little is known about the auditory capabilities of the dunnart, and of marsupials in general. Consequently, this study sought to investigate several electrophysiological and anatomical properties of the dunnart auditory system. Auditory brainstem responses (ABR) were recorded to brief (5ms) tone pips at a range of frequencies $(4-47.5 \mathrm{kHz})$ and intensities to determine auditory brainstem thresholds. The dunnart ABR displayed multiple distinct peaks at all test frequencies, similar to other mammalian species. ABR showed the dunnart is most sensitive to higher frequencies increasing up to $47.5 \mathrm{kHz}$. Morphological observations (Nissl stain) revealed that the auditory structures thought to contribute to the first peaks of the ABR were all distinguishable in the dunnart. Structures identified include the dorsal and ventral subdivisions of the cochlear nucleus, including a cochlear nerve root nucleus as well as several distinct nuclei in the superior olivary complex, such as the medial nucleus of the trapezoid body, lateral superior olive and medial superior olive. This study is the first to show functional and anatomical aspects of the lower part of the auditory system in the Fattailed dunnart. 
1 Physiological and anatomical investigation of the auditory brainstem in the Fat-

2 tailed dunnart (Sminthopsis crassicaudata)

3

4 A. Garrett ${ }^{3,4 *}$, V. Lannigan ${ }^{1,3}$, N. Yates ${ }^{1,5}$, J. Rodger ${ }^{1,3 \#}$, W.H.A.M. Mulders ${ }^{1,2 \#}$

5

$6{ }^{1}$ School of Human Sciences, M311, University of Western Australia, 35 Stirling Highway,

7 Crawley, Western Australia, 6009, Australia.

$8 \quad$ Ear Science Institute Australia, 1/1 Salvado Rd, Subiaco, Western Australia, 6008, Australia.

$9 \quad{ }^{3}$ School of Biological Sciences, University of Western Australia, 35 Stirling Highway, Crawley,

10 Western Australia, 6009, Australia.

$11{ }^{4}$ Department of Systems Neuroscience, JFB Institute of Zoology and Anthropology, Bernstein

12 Focus Neurotechnology (BFNT), Georg-August-Universität Göttingen, Von-Siebold Strasse 6,

13 D-37075 Göttingen, Germany

$14{ }^{5}$ The Queensland Brain Institute, University of Queensland, St Lucia, Queensland, 4072,

15 Australia

16

17 \# These authors have made equal contributions to this paper

19 * Correspondence: Andrew Garrett. Department of Systems Neuroscience, JFB Institute of

20 Zoology and Anthropology, Bernstein Focus Neurotechnology (BFNT), Georg-August-

21 Universität Göttingen, Von-Siebold Strasse 6, D-37075 Göttingen, Germany, email: andrew-

22 richard.garrett@biologie.uni-goettingen.de

24 Author declaration: The authors declare no conflicts of interest related to this manuscript. 
25 Abstract

26 The fat-tailed dunnart (Sminthopsis crassicaudata) is a small (10-20g) native marsupial endemic

27 to the south west of Western Australia. Currently little is known about the auditory capabilities

28 of the dunnart, and of marsupials in general. Consequently, this study sought to investigate

29 several electrophysiological and anatomical properties of the dunnart auditory system. Auditory

30 brainstem responses $(\mathrm{ABR})$ were recorded to brief $(5 \mathrm{~ms})$ tone pips at a range of frequencies (4-

$3147.5 \mathrm{kHz}$ ) and intensities to determine auditory brainstem thresholds. The dunnart ABR

32 displayed multiple distinct peaks at all test frequencies, similar to other mammalian species.

33 ABR showed the dunnart is most sensitive to higher frequencies increasing up to $47.5 \mathrm{kHz}$.

34 Morphological observations (Nissl stain) revealed that the auditory structures thought to contribute to the first peaks of the ABR were all distinguishable in the dunnart. Structures identified include the dorsal and ventral subdivisions of the cochlear nucleus, including a cochlear nerve root nucleus as well as several distinct nuclei in the superior olivary complex, such as the medial nucleus of the trapezoid body, lateral superior olive and medial superior olive. This study is the first to show functional and anatomical aspects of the lower part of the auditory system in the Fat-tailed dunnart. 


\section{Introduction}

Marsupials evolved separately from eutherian mammals in the Cretaceous period and now form a highly diverse group with populations in the Americas and Australia (Luo, Yuan, Meng, \& Ji, 2011; Nilsson et al., 2010). One marsupial, the fat-tailed dunnart (Sminthopsis crassicaudata), is a small (10-20g) insectivorous Australian marsupial (Frey, 1991; Morton, 1978a) that is named after its characteristic swollen tail that contains stored fat (Godfrey, 1968). The fat-tailed dunnart is a solitary animal with a widespread distribution across the southern and western parts of Australia inhabiting a variety of arid environments including open woodland, low scrublands, grasslands on clay or sand soils and farmlands (Morton, 1978a). Within these varied environments, the nocturnal dunnart hunts predominantly insects while itself being preyed upon by other predators such as snakes, feral cats and barn owls (Morton, 1978b)

Interestingly, the visual system in the fat-tailed dunnart has been shown to be different from most other marsupials as well as most eutherian mammals as they are trichromatic (Cowing, Arrese, Davies, Beazley, \& Hunt, 2008; Ebeling, Natoli, \& Hemmi, 2010). Being predominantly nocturnal (Levy, Dayan, Porter, \& Kronfeld-Schor, 2019) the fat-tailed dunnart is likely to also heavily depend on its sense of hearing and its ability to localise sound as a means for prey detection, predator avoidance and species-specific communication (Osugi, Foster, Temple, \& Poling, 2011). Previous work in a range of marsupial families such as northern quoll (Dasyurus hallucatus) (Aitkin, Nelson, \& Shepherd, 1996), brush-tailed possums (Trichosurus vulpecula) (Signal, Foster, \& Temple, 2001), and the tammar wallaby (Macropus eugenii) (Liu, 2003; Liu, Hill, \& Mark, 2001) has shown that the overall structure of the auditory brainstem is largely consistent within eutherian mammals, enabling the distinction of several subnuclei in cochlear nuclei (CN), superior olivary complex (SOC) and inferior colliculus (Aitkin, 1998).

However, the relative size and detailed structure of the subcortical structures in the auditory system is known to be highly varied both in eutherian mammals and marsupials (Glendenning \& Masterton, 1998). For example, the $\mathrm{CN}$ represents about $13 \%$ of the whole auditory system in the swamp wallaby, but approximately $37 \%$ in the pocket gopher. In addition, there exists a large degree of heterogeneity in the anatomical architecture of the $\mathrm{CN}$ and principal nuclei of the SOC (Glendenning \& Masterton, 1998). For example, in some of the Muridae such as rat, mouse and gerbil (López, Merchán, Bajo, \& Saldaña, 1993) as well as in some marsupials (Willard, 1993) 
81 the auditory nerve contains a small group of large neurons, the so-called cochlear nerve root neurons, whereas this does not appear to be the case in for instance cat or guinea pig. In the SOC, the lateral superior olive (LSO) forms a S-shaped segment in many species such as guinea pig, cat and gerbil (Grothe \& Park, 2000) but has been described as a triangle shape in marsupials (Aitkin 1996). In marsupials the cochlear nucleus is located medial to the restiform body, whereas in other mammals the it is found lateral to the restiform body (Aitkin, 1996).

With regard to functional studies, the auditory brainstem response (ABR) has been shown to reveal the typical waveforms (i.e. waves I-V present) between $1-90 \mathrm{kHz}$ with lowest thresholds between 12-16kHz in the short-tailed opossum (Monodelphus domestica) (Reimer, 1996b). Click-evoked ABRs obtained from tammar wallaby also showed typical peaks and the appearance of the peaks during development correlated with the development of the known anatomical substrates of the ABR waves (Liu, 2003; Liu, Hill, \& Mark, 2001).

With the exception of a few references to the striped-faced dunnart (Sminthopsis macroura) by Aitkin (1998) very little is known about the anatomy and physiology of the dunnart auditory system. In view of the fact that the fat-tailed dunnart has specific adaptations in its visual system, this paper explored functional and anatomical aspects of its auditory system to investigate whether this sensory system also has distinct features compared to other marsupials. For this purpose, we combined electrophysiological (ABR) and anatomical (Nissl staining) investigations of the auditory brainstem in the dunnart. For the latter we focussed on cochlear nucleus and the main nuclei in the SOC, known to be involved in sound localization.

\section{Materials and Methods}

\section{Animals}

Eight fat-tailed dunnarts (Sminthopsis crassicaudata) aged between 12 and 18 months (12-18g weight) of either sex were used for this study. Precise age was not known but was estimated based on arrival in the animal facilities, weight and time of experimentation. The animals were separately housed in enriched cages containing running discs, rocks and a covered nest. Food (Science Diet Sensitive Stomach Cat Food supplemented with live crickets and mealworms) and 
109

110

111

112

113

114

115

116

117

118

119

120

121

122

123

124

125

126

127

128

129

130

131

132

133

134

135

136

137

water were supplied ad libitum. The vivariums were maintained at $22^{\circ} \mathrm{C}$ with a 12 -hour Day night cycle. All procedures conformed to NIH guidelines on the use of animals for experimentation (USA) and were approved by the University of Western Australia's Animal Ethic Committee (RA/3/100/1123).

\section{Auditory Brainstem Response Measurements}

The fat-tailed dunnarts were anaesthetised via intraperitoneal injection with ketamine $(75 \mathrm{mg} / \mathrm{kg})$ and medetomidine $(1 \mathrm{mg} / \mathrm{kg})$. Animals were maintained at near physiological temperature $\left(38^{\circ} \mathrm{C}\right)$ using both a heating pad and an ambient room heater for the entirety of the auditory brainstem response (ABR) recording (60-90 minutes per animal). ABRs were measured as previously described (Yates, Robertson, Martin-Iverson, \& Rodger, 2014). In brief, ABRs were recorded in a sound attenuated room and sound stimuli were generated by custom made Neurosound software (M. Lloyd Cambridge) via a RME DIGI 9636 sound card ( $96 \mathrm{kHz}$ sampling rate). Average ABRs ( $\mathrm{n}=400$ stimuli) were evoked using pure tone bursts ( $5 \mathrm{~ms}$ duration, $1 \mathrm{~ms}$ rise-falltime, rate 10/s), delivered to the animal using a plastic cone attached to a reverse driven $1 / 4$ inch condenser microphone (Brüel and Kjær type 4134). The sound-output was calibrated at the level of the eardrum using a Brüel \& Kjær pistonphone (94dB SPL at 1000Hz) (Yates et al., 2014). The acoustic coupler was placed using a surgical microscope to touch the lower edge of the left tragus and was directed towards external auditory meatus. During the course of the experiments, we observed no movement of the animal or auditory coupler.

ABR responses were recorded via an insulated silver-wire electrode inserted subdermally at the vertex. A reference electrode was placed above the left mastoid at the base of the pinna and a ground electrode was inserted into the tail. Differential recordings were made using an AC coupled amplifier (DAM50, World Precision Instruments) with a gain of 1000x and band pass filtering at (300-3000Hz). Average ABR responses were sampled by Powerlab/4ST (AD Instruments) and stored for offline analyses.

ABR thresholds were determined at 4, 8, 16, 24, 32 and $47.5 \mathrm{kHz}$. In view of the sampling rate of our sound card $47.5 \mathrm{kHz}$ was the maximum frequency tested. Each sound stimulus was presented first at $10 \mathrm{~dB}$ attenuation followed by sound intensities decreasing in $10 \mathrm{~dB}$ increments 
138 until after the disappearance of overt ABR peaks (I and V) in the recording. Upon disappearance 139 of the $\mathrm{ABR}$, the sound intensity was increased in $5 \mathrm{~dB}$ steps until the visual reappearance of the 140 peaks in the waveform. Sound stimuli were converted into sound pressure (SPL, re $20 \mu \mathrm{Pa}$ ) levels 141 using a Bruel and Kjaer pistonphone (94dB SPL at 1000Hz). ABR traces were analyzed using 142 AxoGraph X V1.5.0 (J. Clements, Australia) and thresholds were determined by visual 143 inspection. ABR threshold was estimated as the lowest intensity where peaks I and V could still 144 be identified. The threshold estimation procedure employed here, was undertaken by 3 different 145 observers and yielded consistent estimates (less than $5 \mathrm{~dB}$ difference).

Histological preparation

Dunnarts were terminally anaesthetised with $0.2 \mathrm{ml}$ euthal (pentobarbitone sodium $170 \mathrm{mg} / \mathrm{mL}$, phenytoin sodium $25 \mathrm{mg} / \mathrm{mL}$ ). Animals were then perfused with saline $(0.9 \%)$ followed by paraformaldehyde ( $4 \%$ in $0.1 \mathrm{M}$ phosphate buffered saline, PBS). Regions of brainstem containing auditory nuclei were removed and cryoprotected ( $30 \%$ sucrose in $0.1 \mathrm{M}$ PBS for $24 \mathrm{~h}$ ) and sectioned at $30 \mu \mathrm{m}$ using a cryostat (Leica CM1900).

For cresyl violet staining, horizontal sections were washed with PBS for four minutes and then dehydrated in graded ethanol solutions ( $70 \%-95 \%$, one minute). Slides were heated in a microwave for 2 minutes in a $500 \mathrm{~mL}$ solution of $95 \%$ ethanol and $5 \%$ glacial acetic acid (Sigma), followed by rehydration in descending ethanol solutions ( $95 \%$ to $70 \%, 20$ seconds each) and washed in PBS for one minute. Sections were then placed in warmed cresyl violet solution ( $0.5 \%$ cresyl violet) for eight minutes. After staining, sections were rapidly exchanged through ascending ethanol solutions (70\%-95\%, 15 seconds each) and differentiated at room temperature in $95 \%$ ethanol and 5\% acetic acid for 5 minutes. Finally, slides were washed with three $100 \%$ ethanol and cleared in xylene. Slides were cover-slipped with DePeX (ProSciTech) mounting media and dried overnight prior to microscopy. 
166

167

168

169

170

171

172

173

174

175

176

177

178

179

180

181

182

183

184

185

186

187

188

189

190

191

192

\section{Microscopy and analysis}

Images of cresyl violet stained sections were captured using an Olympus DP70 camera and DP Controller (Olympus Corporation, image size 4080x3072pixels). High-power micrographs were captured using a Nikon DS-U2/L2 camera with NIS-Elements (Nikon AR 3.0, image size 2560x1920pixels). Using standard anatomical markers such as neuronal shape, neuronal density, and somatic alignment, the auditory nuclei (CN and SOC) were identified in the dunnart. Nuclei were observed under low power to determine the area and extent of the nucleus. Images for publication underwent minor adjustments in brightness and contrast.

\section{Results}

Auditory Brainstem Response

A typical ABR was observed in the fat-tailed dunnart (figure 1). At moderate to high sound intensities, the ABR showed five distinct peaks within the first $6 \mathrm{~ms}$ after onset of the tone stimuli. ABRs were evoked at all frequencies tested in this study (between 4 and $47.5 \mathrm{kHz}$ ). ABR threshold was estimated as the lowest intensity where peak I and V could still be identified (typical example at $47.5 \mathrm{kHz}$ shown in figure $2 \mathrm{a})$. Average thresholds $(\mathrm{n}=6-8)$ depicted as audiograms (figure $2 \mathrm{~b}$ ) reveal the fat-tailed dunnart ABR is more sensitive (lower thresholds) with increasing frequency. Currently however, it cannot be established whether $47.5 \mathrm{kHz}$ is the most sensitive frequency or if $\mathrm{ABR}$ thresholds decline rapidly at higher frequencies.

In agreement with the known characteristics of ABR responses, peak I amplitudes increased with increasing sound intensity (figure 2c). Similarly, increasing sound intensities resulted in a shortening of ABR latencies (data for 4,24 and $47.5 \mathrm{kHz}$ shown in figure $2 \mathrm{~d}$ ).

\section{Histological analysis}

\section{The cochlear nerve root and cochlear nuclei}

Similar to other known marsupial species such as the brush-tailed possum and quoll, the cochlear nuclei $(\mathrm{CN})$ reside medial to the restiform body ( $\mathrm{rb}$ in figure $3 \mathrm{a}-\mathrm{c})$. The ventral cochlear nucleus 
193 (VCN) as a whole is clearly identifiable in the dunnart (figure 3c,h) with round small closely 194 packed cells of the anteroventral cochlear nucleus (AVCN) in rostral levels to the dorsal cochlear

195

196 197

198 199

200

201

202

203

204

205

206

207

208

209

210

211

212

213

214

215

216

217

218

219

220 nucleus (DCN). A sparsely populated posteroventral cochlear nucleus (PVCN) containing large nuclei was observed in more caudal sections containing the other cochlear nuclei (figure $3 c, d, e, f)$.

On gross appearance, the dunnart DCN is a trigonal shaped nucleus. In more caudal sections, a prominent tri-laminar DCN could clearly be subdivided into a molecular (I in figure 3f), fusiform layer (II in figure $3 \mathrm{f}$ ) and polymorphic layers (III in figure $3 \mathrm{f}$ ). The DCN was bounded laterally by the small cell cap layer (scc, figure $3 \mathrm{~d}, \mathrm{f}$ ).

Briefly. the dunnart also shows a clearly defined cochlear nerve root nucleus (CNR) (figure 3e and g), consisting of large neurons clustered within the passing nerve fascicles.

\section{The superior olivary complex nuclei}

The nuclei of the superior olivary complex (SOC) in the dunnart closely resembled their anatomical correlates found in eutherian mammals. Of the three principal SOC nuclei lateral superior olive (LSO), medial superior olive (MSO), and the medial nucleus of the trapezoid body (MNTB), the most prominent and distinguishable nucleus in the dunnart was the MNTB (figure 4a,b). The MNTB occupied a familiar position within the brainstem and the cells of the MNTB were not densely packed presumably due to their location within the passing trapezoid body projection (see figure $4 \mathrm{~b}$ ). A small MSO (typically observed within one to two histological sections) was observed as a linear cluster of pleiomorphic cells aligned along a dorsal-ventral axis (figure 4a-c).

The lateral superior olive (LSO) of the dunnart was not as well defined as found in similarly sized eutherian species (figure 4c). Despite this, the LSO was observed as a round nucleus located near the latero-ventral surface of the brainstem in transverse sections often containing the MNTB. The LSO could be subdivided into densely stained elongated cells in more marginal areas (figure $4 \mathrm{c}, 1 \mathrm{so}(\mathrm{m})$ ), whereas lightly stained bipolar nuclei were found to occupy more core or central locations (figure $4 \mathrm{c}$, 1so(c)). 
221 A nucleus corresponding to superior paraolivary nucleus (SPN, figure 4 a-c), was observed

222 residing dorsomedially between the MNTB and the MSO. Somata in this region contained large

223 densely stained multipolar cells with no clear systematic orientation.

\section{Discussion}

227

228

229

230

231

232

233

234

235

236

237

238

239

240

241

242

243

244

245

246

247

248

Here we characterise some of the anatomical and electrophysiological features of the ascending auditory pathway in the fat-tailed dunnart. With the exception of Aitkin (1998), there has been very little characterisation of the dunnart auditory system, therefore we sought to establish normative values of the fat-tailed dunnart auditory system. In addition to identifying common auditory nuclei, we found that the anesthetised fat-tailed dunnart auditory system is remarkably sensitive to high frequency stimuli.

The ABR represents the average response to repetitive sound stimuli of neuronal populations in the auditory pathway. Waveform analysis of the ABR revealed 5 definite peaks (Reimer, 1996) with short latency, corresponding to the action-potential volleys from the auditory nerve through to inferior colliculus (Liu et al., 2001). In the current study, not only were we still able to evoke ABR responses to high frequency stimuli $(47.5 \mathrm{kHz})$, but $\mathrm{ABR}$ thresholds improved at high frequencies. These ABR findings are puzzling and present a contrast to the only previously published data from a dunnart species (Sminthopsis macroaura), which displayed a frequency range of $1-40 \mathrm{kHz}$ and a minimum, or best threshold at 10kHz (Aitkin, 1998). However, this study was limited by low animal numbers $(n=2)$ and lack of detail in the methodology, making it unclear whether $40 \mathrm{kHz}$ was the highest frequency attempted.

Nonetheless, high frequency sensitivity is quite common in small non-echolocating mammals such as the leaf-eared mouse and spiny mouse (Heffner, Koay, \& Heffner, 2001). In fact, upon closer inspection of cochlear and ABR audiograms taken from several rodent species including the mouse (Mus musculus), a second local minimum is present (20-30dB SPL) at around $50 \mathrm{kHz}$ (Ehret, 1976; Heffner et al., 2001), and similarly, secondary local minima are also found in echolocating mammals ( $\sim 15 \mathrm{~dB}$ SPL at $>45 \mathrm{kHz})$ (Koay, Heffner, \& Heffner, 1998). 
249 With the exception of the cat (Felis catus), animals with smaller head sizes have small functional

250 interaural distances and tend to have higher audible frequencies (Heffner et al., 2001; Koay et al., 251 1998). In agreement with this, another marsupial, the northern quoll (Dasyurus hallucatus)

252 which is larger than the dunnart (adults $400 \mathrm{~g}, 5 \mathrm{~cm}$ snout-ear), is most sensitive at $10 \mathrm{kHz}(10 \mathrm{~dB}$

253 SPL) with rapid loss of sensitivities at 40kHz (50-80dB SPL) (Aitkin, Nelson, \& Shepherd,

254 1994; Oakwood, 2002). Similarly, the Brazilian short-tailed opossum (Monodelphis domestica)

255 also a marsupial larger than the fat-tailed dunnart (rat-size) shows best thresholds between 8 and $25612 \mathrm{kHz}$ (20 dB SPL) and an upper audible frequency limit of 60kHz (Reimer, 1995). Therefore,

257 given its small size (12-18g), the high frequency sensitivity observed in the fat-tailed dunnart

258 may be in line with its size, but conflicts with the limited data from the stripe faced dunnart

259 (Aitkin, 1998), which is of similar size. Therefore, we cannot exclude the possibility that this

260 audiogram of the fat-tailed dunnart represents a specific adaptation to its auditory environment,

261 in line with the specific adaptation found in its visual system (Cowing et al., 2008; Ebeling et al.,

262 2010). The reasons for such specialised adaptations within its sensory system remain unclear. As

263 discussed in Ebeling et al. it may represent specific adaptations to the visual and auditory

264 ecology or, alternatively, adaptations in early ancestors (Ebeling et al., 2010).

265 The anatomy of the auditory brainstem in the fat-tailed dunnart reveals a similar pattern of 266 auditory nuclei as reported previously across a range of marsupials (Aitkin, 1998). The CNR is

267 present in many small marsupials including the yellow-bellied glider (Petaurus australis),

268 Northern quoll (Aitkin, Byers, \& Nelson, 1986) but also in muridae (López et al., 1993;

269 Merchan, Collia, Lopez, \& Saldana, 1988) While neurons in the CNR nucleus are considered as

270 an extension of the ventral cochlear nucleus (Osen, Lopez, Slyngstad, Ottersen, \& Storm-

271 Mathisen, 1991), it projects to motor components of the pontine reticular and facial nuclei

272 (Lopez, Saldana, Nodal, Merchan, \& Warr, 1999). Although few in number, neurons in the CNR

273 nucleus in the rat respond to sound and thus likely represent an initial auditory nucleus (Sinex,

274 Lopez, \& Warr, 2001). Given its early position within the auditory pathway, sensitivity to sound,

275 and efferent projections to the pontine motor nuclei, the CNR nucleus is thought to play a role in

276 sensorimotor control of acoustic startle responses (Lee, Lopez, Meloni, \& Davis, 1996).

277 The auditory cochlear nuclei in the dunnart were similar in location to other marsupial species

278 studied such as the brush-tailed possum (Trichosurus vulpecula) (Aitkin \& Kenyon, 1981), 
279 multiple glider species (Aitkin, 1996), and northern quoll (Aitkin et al., 1986). Also in agreement 280 with other marsupials, the fat-tailed dunnart's trilaminar DCN was larger than the VCN (Aitkin, 281 1996, 1998). Despite widespread variation across mammalian species (Glendenning \& 282 Masterton, 1998; Illing, Kraus, \& Michler, 2000), the organisation of the SOC was again largely 283 consistent with previous reports. In common laboratory rodents, the three main SOC (LSO, MSO 284 and MNTB) as well as the SPN, are known targets of the cochlear nuclei and it is likely that a 285 similar connectivity exists in marsupials (Aitkin et al., 1986; Bazwinsky-Wutschke, Hartig, Kretzschmar, \& Rubsamen, 2016; Schofield, 1995). The presence of the MSO is not surprising 288 as it is known to persist in almost all mammalian species including the mouse (Fischl et al., 2016; Ollo \& Schwartz, 1979). Furthermore, the location and appearance of the MSO as a linear nucleus in the dunnart is consistent with previous reports in arboreal marsupials (Aitkin, 1996). The MSO is involved in detecting interaural timing differences related to sound localization of lower frequencies (Grothe \& Sanes, 1994). Therefore, it is likely that the functional role of the MSO in these small animals with high frequency sensitivity is relatively limited (Grothe \& Pecka, 2014) and hence its small size in the fat-tailed dunnart is as expected. The LSO and MNTB, involved in detection of higher frequencies based on interaural level differences (Caird \& Klinke, 1983; Grothe \& Koch, 2011) were both present in the fat-tailed dunnart in line with its high frequency sensitivity.

297

298

299

300

301

302

303

304

305

306 307

The relative size of the MNTB is known to vary between species, its relative size being about 5\% of the subcortical auditory system in kangaroo rat and less than $1 \%$ in humans (Glendenning \& Masterton, 1998). In addition, a study by Hilbig et al comparing different primates, showed a marked reduction in MNTB size from macaque to human (Hilbig, Beil, Hilbig, Call, \& Bidmon, 2009). The MNTB in the fat-tailed dunnart was clearly distinguishable with large neurons comparable to the anatomy in rat (Reuss, Disque-Kaiser, De Liz, Ruffer, \& Riemann, 1999). Taken together, the presence of the large well defined MNTB, which is known to provide powerful and precisely-timed glycinergic inhibition to the ipsilateral LSO, MSO and SPN (Adams \& Mugnaini, 1990; Sanes \& Friauf, 2000), completes known anatomical circuits involved with sound-localisation in many eutherian species (Kapfer, Seidl, Schweizer, \& Grothe, 2002; Sanes \& Friauf, 2000; Tollin, 2003). 
308 The LSO is often described as an S-shaped or horseshoe shaped nucleus in many species such as 309 guinea pig, cat and gerbil (Grothe \& Park, 2000). In contrast to the mouse (Ollo \& Schwartz, 310 1979), a distinct shape could not be observed in our histological material. Rather the LSO

311 boundary in the dunnart remained diffuse, in line with the description of Aitkin (1996) in some 312 arboreal marsupials (Aitkin, 1996). Despite the lack of a clear boundary, the LSO did contain 313 subdivisions (marginal and core) which has been reported previously by Willard \& Martin 314 (1983) in the opossum (Willard \& Martin, 1983).

315 While the presence of $\mathrm{CN}$ and SOC in the dunnart suggests an ability to process incoming 316 auditory information particularly in terms of sound localisation, further investigations into the 317 synaptic morphology, neurochemistry and electrophysiology would further help to refine our 318 understanding of the roles these nuclei play within the dunnart and their environment.

\section{Conclusions}

320

321

322

323

324

325

326

327

1

Here we show that the fat-tailed dunnart is an animal species that displays a remarkable high frequency sensitivity. In addition, the auditory brainstem nuclei reveal a large and well developed $\mathrm{CN}$ as well as a MNTB. These nuclei are important in early binaural auditory processing and sound localisation, and their presence in the dunnart suggests similar processing capabilities. In addition to extending the ABR audiograms to higher frequencies, it would be of immediate interest to determine how the hearing sensitivities correspond to species specific communication as well as predator / prey detection and avoidance. (Aitkin et al., 1994). In light of recent reports on the role of the DCN in the analysis of vocalisations (Roberts \& Portfors, 2015), it would be of interest to determine if the DCN performs a similar role in the marsupial.

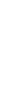




\section{References:}

Adams, J. C., \& Mugnaini, E. (1990). Immunocytochemical evidence for inhibitory and disinhibitory circuits in the superior olive. Hear Res, 49(1-3), 281-298.

Aitkin, L. (1996). The anatomy of the cochlear nuclei and superior olivary complex of arboreal Australian marsupials. Brain Behav Evol, 48(2), 103-114. doi:10.1159/000113189

Aitkin, L. (1998). Hearing, the brain, and auditory communication in marsupials (Vol. 38). Berlin Heidelberg: Springer-Verlag.

Aitkin, L., Byers, M., \& Nelson, J. (1986). Brain stem auditory nuclei and their connections in a carnivorous marsupial, the northern native cat (Dasyurus hallucatus). Brain Behav Evol, 29(1-2), 1-16.

Aitkin, L., \& Kenyon, C. (1981). The auditory brain stem of a marsupial. Brain Behav Evol, 19(3-4), 126143.

Aitkin, L., Nelson, J., \& Shepherd, R. (1994). Hearing, vocalization and the external ear of a marsupial, the northern Quoll, Dasyurus hallucatus. J Comp Neurol, 349(3), 377-388. doi:10.1002/cne.903490305

Bazwinsky-Wutschke, I., Hartig, W., Kretzschmar, R., \& Rubsamen, R. (2016). Differential morphology of the superior olivary complex of Meriones unguiculatus and Monodelphis domestica revealed by calcium-binding proteins. Brain Struct Funct, 221(9), 4505-4523. doi:10.1007/s00429-015-1181-x

Caird, D., \& Klinke, R. (1983). Processing of binaural stimuli by cat superior olivary complex neurons. Exp Brain Res, 52(3), 385-399.

Cowing, J. A., Arrese, C. A., Davies, W. L., Beazley, L. D., \& Hunt, D. M. (2008). Cone visual pigments in two marsupial species: the fat-tailed dunnart (Sminthopsis crassicaudata) and the honey possum (Tarsipes rostratus). Proc Biol Sci, 275(1642), 1491-1499. doi:10.1098/rspb.2008.0248

Ebeling, W., Natoli, R. C., \& Hemmi, J. M. (2010). Diversity of color vision: not all Australian marsupials are trichromatic. PLoS One, 5(12), e14231. doi:10.1371/journal.pone.0014231

Ehret, G. (1976). Development of absolute auditory thresholds in the house mouse (Mus musculus). J Am Audiol Soc, 1(5), 179-184.

Fischl, M., Burger, R., Schmidt-Pauly, M., Alexandrova, O., Sinclair, J., Grothe, B., . . Kopp-Scheinpflug, C. (2016). Physiology and anatomy of neurons in the medial superior olive of the mouse. $J$ Neurophysiol, 116(6), 2676-2688. doi:10.1152/jn.00523.2016

Frey, H. (1991). Energetic Significance of Torpor and Other Energy-conserving Mechanisms in Free-living Sminthopsis crassicaudata (Marsupialia : Dasyuridae) Aust J Zool, 39, 689-708.

Glendenning, K., \& Masterton, R. (1998). Comparative morphometry of mammalian central auditory systems: variation in nuclei and form of the ascending system. Brain Behav Evol, 51(2), 59-89.

Godfrey, G. (1968). Body-Temperature and Torpor in Sminthopsis Crassicaudata and S Larapinta (Marsupialia-Dasyuridae). Journal of Zoology, 156, 499.

Grothe, B., \& Koch, U. (2011). Dynamics of binaural processing in the mammalian sound localization pathway--the role of GABA(B) receptors. Hear Res, 279(1-2), 43-50. doi:10.1016/j.heares.2011.03.013

Grothe, B., \& Park, T. J. (2000). Structure and function of the bat superior olivary complex. Microsc Res Tech, 51(4), 382-402. doi:10.1002/1097-0029(20001115)51:4<382::AID-JEMT7>3.0.CO;2-7

Grothe, B., \& Pecka, M. (2014). The natural history of sound localization in mammals--a story of neuronal inhibition. Front Neural Circuits, 8, 116. doi:10.3389/fncir.2014.00116

Grothe, B., \& Sanes, D. H. (1994). Synaptic inhibition influences the temporal coding properties of medial superior olivary neurons: an in vitro study. J Neurosci, 14(3 Pt 2), 1701-1709. 
380

381

382

383

384

385

386

387

388

389

390

391

392

393

394

395

396

397

398

399

400

401

402

403

404

405

406

407

408

409

410

411

412

413

414

415

416

417

418

419

420

421

422

423

424

425

426

Heffner, R., Koay, G., \& Heffner, H. (2001). Audiograms of five species of rodents: implications for the evolution of hearing and the perception of pitch. Hear Res, 157(1-2), 138-152.

Hilbig, H., Beil, B., Hilbig, H., Call, J., \& Bidmon, H. J. (2009). Superior olivary complex organization and cytoarchitecture may be correlated with function and catarrhine primate phylogeny. Brain Struct Funct, 213(4-5), 489-497. doi:10.1007/s00429-008-0201-5

Illing, R., Kraus, K., \& Michler, S. (2000). Plasticity of the superior olivary complex. Microsc Res Tech, 51(4), 364-381. doi:10.1002/1097-0029(20001115)51:4<364::AID-JEMT6>3.0.CO;2-E

Kapfer, C., Seidl, A. H., Schweizer, H., \& Grothe, B. (2002). Experience-dependent refinement of inhibitory inputs to auditory coincidence-detector neurons. Nat Neurosci, 5(3), 247-253. doi:10.1038/nn810

Koay, G., Heffner, R., \& Heffner, H. (1998). Hearing in a megachiropteran fruit bat (Rousettus aegyptiacus). J Comp Psychol, 112(4), 371-382.

Lee, Y., Lopez, D., Meloni, E., \& Davis, M. (1996). A primary acoustic startle pathway: obligatory role of cochlear root neurons and the nucleus reticularis pontis caudalis. J Neurosci, 16(11), 3775-3789.

Levy, O., Dayan, T., Porter, W. P., \& Kronfeld-Schor, N. (2019). Time and ecological resilience: can diurnal animals compensate for climate change by shifting to nocturnal activity? Ecological Monographs, 89(1), e01334.

Liu, G. (2003). Functional development of the auditory brainstem in the tammar wallaby (Macropus eugenii): the superior olivary complex and its relationship with the auditory brainstem response (ABR). Hear Res, 175(1-2), 152-164.

Liu, G., Hill, K., \& Mark, R. (2001). Temporal relationship between the auditory brainstem response and focal responses of auditory nerve root and cochlear nucleus during development in the tammar wallaby (Macropus eugenii). Audiol Neurootol, 6(3), 140-153. doi:10.1159/000046821

López, D. E., Merchán, M. A., Bajo, V. M., \& Saldaña, E. (1993). The Cochlear Root Neurons in the Rat, Mouse and Gerbil. In M. A. Merchán, J. M. Juiz, D. A. Godfrey, \& E. Mugnaini (Eds.), The Mammalian Cochlear Nuclei: Organization and Function (pp. 291-301). Boston, MA: Springer US.

Lopez, D. E., Saldana, E., Nodal, F. R., Merchan, M. A., \& Warr, W. B. (1999). Projections of cochlear root neurons, sentinels of the rat auditory pathway. J Comp Neurol, 415(2), 160-174.

Luo, Z. X., Yuan, C. X., Meng, Q. J., \& Ji, Q. (2011). A Jurassic eutherian mammal and divergence of marsupials and placentals. Nature, 476(7361), 442-445. doi:10.1038/nature10291

Merchan, M. A., Collia, F., Lopez, D. E., \& Saldana, E. (1988). Morphology of cochlear root neurons in the rat. J Neurocytol, 17(5), 711-725.

Morton, S. R. (1978a). Ecological Study of Sminthopsis-Crassicaudata (Marsupialia Dasyurdae).1. Distribution, Study Areas and Methods. Australian Wildlife Research, 5(2), 151-162.

Morton, S. R. (1978b). Ecological Study of Sminthopsis-Crassicaudata (Marsupialia Dasyuridae). 2. Behavior and Social-Organization. Australian Wildlife Research, 5(2), 163-182.

Nilsson, M. A., Churakov, G., Sommer, M., Tran, N. V., Zemann, A., Brosius, J., \& Schmitz, J. (2010). Tracking marsupial evolution using archaic genomic retroposon insertions. PLoS Biol, 8(7), e1000436. doi:10.1371/journal.pbio.1000436

Oakwood, M. (2002). Spatial and social organization of a carnivorous marsupial Dasyurus hallucatus (Marsupialia: Dasyuridae). J Zool Lond, 257, 237-248.

Ollo, C., \& Schwartz, I. R. (1979). The superior olivary complex in C57BL/6 mice. Am J Anat, 155(3), 349373. doi:10.1002/aja.1001550306

Osen, K. K., Lopez, D. E., Slyngstad, T. A., Ottersen, O. P., \& Storm-Mathisen, J. (1991). GABA-like and glycine-like immunoreactivities of the cochlear root nucleus in rat. J Neurocytol, 20(1), 17-25.

Osugi, M., Foster, T. M., Temple, W., \& Poling, A. (2011). Behavior-based assessment of the auditory abilities of brushtail possums. J Exp Anal Behav, 96(1), 123-138. doi:10.1901/jeab.2011.96-123

Peer] reviewing PDF | (2019:04:36946:1:1:NEW 22 Aug 2019) 
427

428

429

430

431

432

433

434

435

436

437

438

439

440

441

442

443

444

445

446

447

448

449

450

451

452

453

454

455

456

457

458

459

460

461
Reimer, K. (1995). Hearing in the marsupial Monodelphis domestica as determined by auditory-evoked brainstem responses. Audiology, 34(6), 334-342.

Reimer, K. (1996). Characterization of the brainstem auditory evoked potential (BAEP) in the grey shorttailed opossum (Monodelphis domestica). Audiology, 35(4), 204-216.

Reuss, S., Disque-Kaiser, U., De Liz, S., Ruffer, M., \& Riemann, R. (1999). Immunfluorescence study of neuropeptides in identified neurons of the rat auditory superior olivary complex. Cell Tissue Res, 297(1), 13-21.

Roberts, P. D., \& Portfors, C. V. (2015). Responses to Social Vocalizations in the Dorsal Cochlear Nucleus of Mice. Front Syst Neurosci, 9, 172. doi:10.3389/fnsys.2015.00172

Sanes, D. H., \& Friauf, E. (2000). Development and influence of inhibition in the lateral superior olivary nucleus. Hear Res, 147(1-2), 46-58.

Schofield, B. R. (1995). Projections from the cochlear nucleus to the superior paraolivary nucleus in guinea pigs. J Comp Neurol, 360(1), 135-149. doi:10.1002/cne.903600110

Sinex, D. G., Lopez, D. E., \& Warr, W. B. (2001). Electrophysiological responses of cochlear root neurons. Hear Res, 158(1-2), 28-38.

Tollin, D. J. (2003). The lateral superior olive: a functional role in sound source localization. Neuroscientist, 9(2), 127-143. doi:10.1177/1073858403252228

Willard, F. H. (1993). Postnatal Development of Auditory Nerve Projections to the Cochlear Nucleus in Monodelphis Domestica. In M. A. Merchán, J. M. Juiz, D. A. Godfrey, \& E. Mugnaini (Eds.), The Mammalian Cochlear Nuclei: Organization and Function (pp. 29-42). Boston, MA: Springer US.

Willard, F. H., \& Martin, G. F. (1983). The auditory brainstem nuclei and some of their projections to the inferior colliculus in the North American opossum. Neuroscience, 10(4), 1203-1232. doi:10.1016/0306-4522(83)90109-4

Yates, N., Robertson, D., Martin-Iverson, M., \& Rodger, J. (2014). Auditory brainstem responses of ephrin-A2, ephrin-A5(-/-) and ephrin-A2A5(-/-) mice. Audiol Neurootol, 19(2), 115-126.

doi:10.1159/000357029 


\section{$462 \quad$ Figure legends}

463 Figure 1. Characteristic ABR recording from the fat-tailed dunnart (tone burst indicated with 464 black bar below the graph, $47.5 \mathrm{kHz}, 5 \mathrm{~ms}$ duration, $52 \mathrm{~dB}$ SPL). Grey line represents the 465 background noise from the recording equipment. Main peaks of ABR indicated by roman 466 numerals and accompanied by abbreviated corresponding auditory nuclei. AN - auditory nerve, 467 CV- cochlear nuclei, SOC - superior olivary nuclei, LL - lateral lemniscus, IC- inferior 468 colliculus.

Figure 2. ABR thresholds in fat-tailed dunnart. (a): ABR recordings at 6 different intensities (42, $32,22,12,7$ and $2 \mathrm{~dB}$ SPL indicated right of waveforms) in response to a $47.5 \mathrm{kHz}$ tone burst. Waveform corresponding to ABR threshold (disappearance of peaks I and V - shown by asterisks at top) is shown in thick black line. Black bar underneath waveforms indicates duration of tone burst. (b): Audiogram showing ABR thresholds at different frequencies. Individual animal thresholds are shown in grey with numbers in brackets above each point indicate number of animals per data point. Due to several animals with the same thresholds, the number of individual response points, may not appear to correspond with the number of animal in parenthesis (c): Input-output function of the peak I amplitude at 4, 24 and $47.5 \mathrm{kHz}$. (d) Inputoutput function of the latency of peak I at 4, 24 and $47.5 \mathrm{kHz}$. Each data point shows mean \pm SEM. N.B. in panel (c) and (d) some of the points at very low sound intensity are the values derived from 1 or 2 animals.

Figure 3. Overview of the fat-tailed dunnart auditory brainstem. Nissl staining of transverse sections reveals prominent auditory nerve root nucleus and cochlear nuclei. Images are organised caudal to rostral. The dorsal cochlear nucleus resides medio-dorsal to the restiform body in the caudal regions (shown in a, with high power image in b). (c and d): More rostrally the ventral cochlear nucleus shows prominently as well. (d), (e), and (f): further rostral the trilaminar arrangement of the dorsal cochlear nucleus is clearly visible (f) as well as the cochlear nerve root nucleus (g). At more rostral level $(\mathrm{h})$ the ventral cochlear nucleus shows a separation between 
491 in b,d and f,g. Distance between panel a and c: $240 \mu \mathrm{m}$, between c and e $90 \mu \mathrm{m}$, and between e 492 and $\mathrm{h} 210 \mu \mathrm{m}$. Section coordinates in panel (h) are for all sections. Abbreviations: cnr - cochlear 493 nerve root, $\mathrm{cb}$ - cerebellum, dcn - dorsal cochlear nucleus, fn - facial nucleus, rb - restiform 494 body, avcn - anteroventral cochlear nucleus, pven- posteroventral cochlear nucleus, in panel f: I 495 - molecular later II - fusiform layer III - polymorphic layer

496

497 Figure 4. Transverse sections of the superior olivary complex (SOC) nuclei in the fat-tailed 498 dunnart. The four main nuclei evident include the medial nucleus of the trapezoid body (MNTB) 499 ( $a$ and $b$ ) residing within the fibres of the trapezoid body ( $t b$ marked in a). The superior 500 paraolivary nucleus (SPN) is located dorsomedial to the MNTB. Located laterally to the MNTB 501 and SPN is the linear medial superior olive (MSO) (a, b with outline in c). The lateral superior 502 olive (LSO) (outline in c) can be seen lateral to the MSO and contains a marginal (lso-m) and 503 core (lso-c) regions (outlines in a, c). The boundary of the LSO shown in panel c is tentative and 504 derive from alignment of neuronal somata. Micrographs are taken at 2x (a) and 10x (b). Section 505 coordinates in panel (c) are for all sections. Scale bars denote $1 \mathrm{~mm}$ in a and $200 \mu \mathrm{m}$ in b, c. 506 Abbreviations: 1so - lateral superior olive, mntb - medial nucleus of the trapezoid body, mso 507 medial superior olive, spn- superior paraolivary nucleus, tb - trapezoid body. 
Figure 1

Characteristic $A B R$ recording from the dat-tailed dunnart (tone burst indicated with black bar below the graph, $47.5 \mathrm{kHz}, 5 \mathrm{~ms}$ duration, 52dB SPL).

Grey line represents the background noise from the recording equipment. Main peaks of $A B R$ indicated by roman numerals and accompanied by abbreviated corresponding auditory nuclei. AN - auditory nerve, CV- cochlear nuclei, SOC - superior olivary nuclei, LL - lateral lemniscus, IC- inferior colliculus.
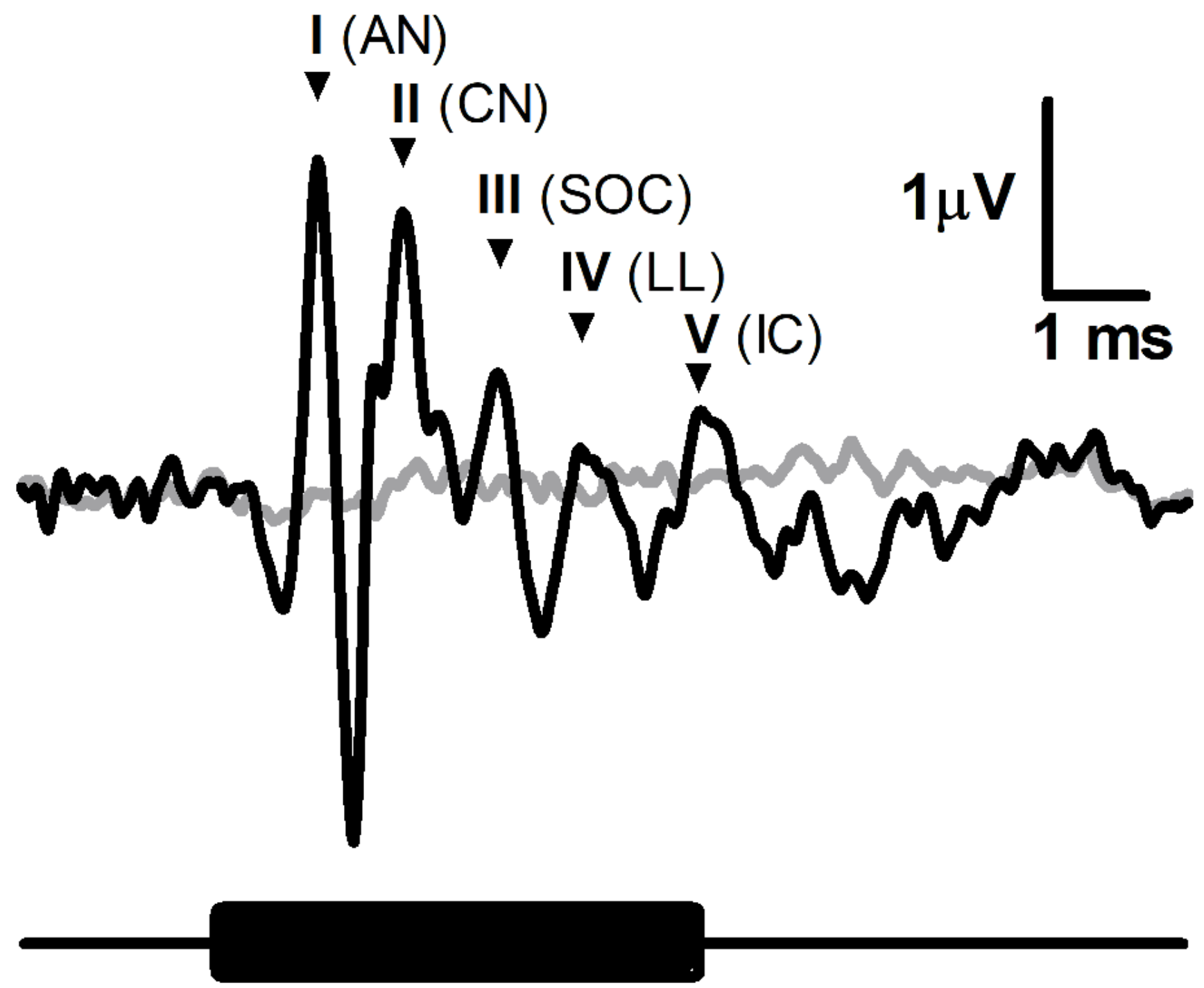


\section{Figure 2}

ABR thresholds in fat-tailed dunnart.

(a): ABR recordings at 6 different intensities $(42,32,22,12,7$ and $2 \mathrm{~dB}$ SPL indicated right of waveforms) in response to a $47.5 \mathrm{kHz}$ tone burst. Waveform corresponding to ABR threshold (disappearance of peaks I and V - shown by asterisks at top) is shown in thick black line. Black bar underneath waveforms indicates duration of tone burst. (b): Audiogram showing $A B R$ thresholds at different frequencies. Individual animal thresholds are shown in grey with numbers in brackets above each point indicate number of animals per data point. Due to several animals with the same thresholds, the number of individual response points, may not appear to correspond with the number of animal in parenthesis (c): Input-output function of the peak I amplitude at 4, 24 and $47.5 \mathrm{kHz}$. (d) Input-output function of the latency of peak I at 4, 24 and $47.5 \mathrm{kHz}$. Each data point shows mean \pm SEM. N.B. in panel (c) and (d) some of the points at very low sound intensity are the values derived from 1 or 2 animals. 
a

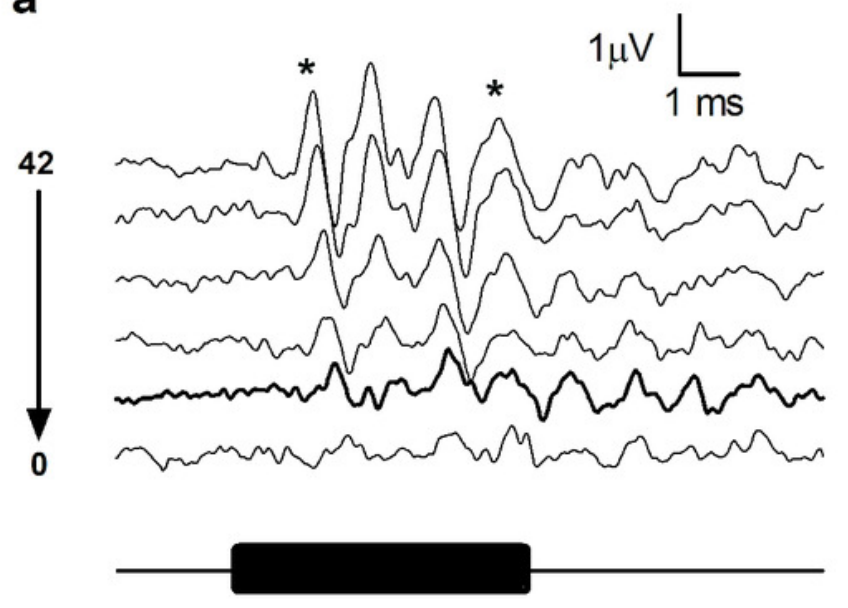

C

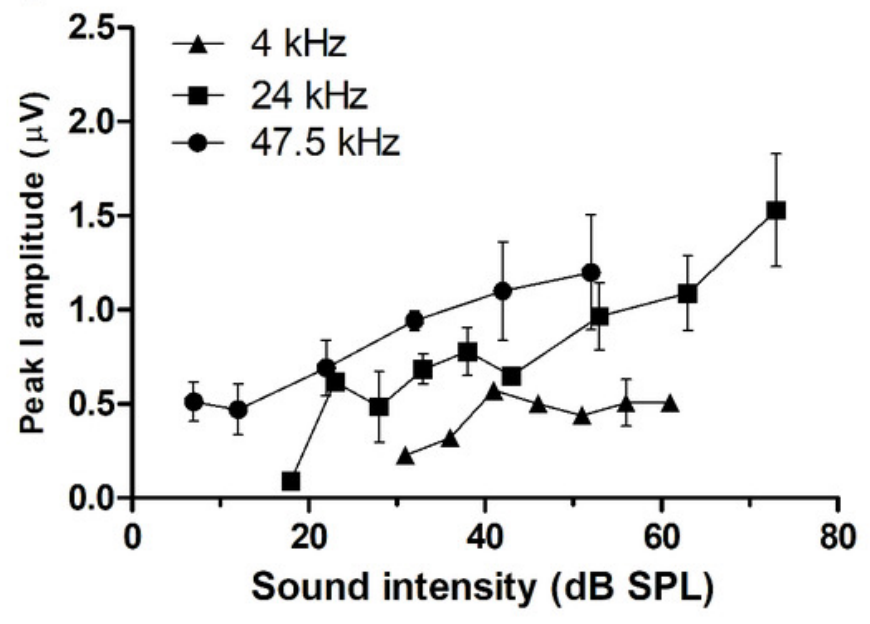

b

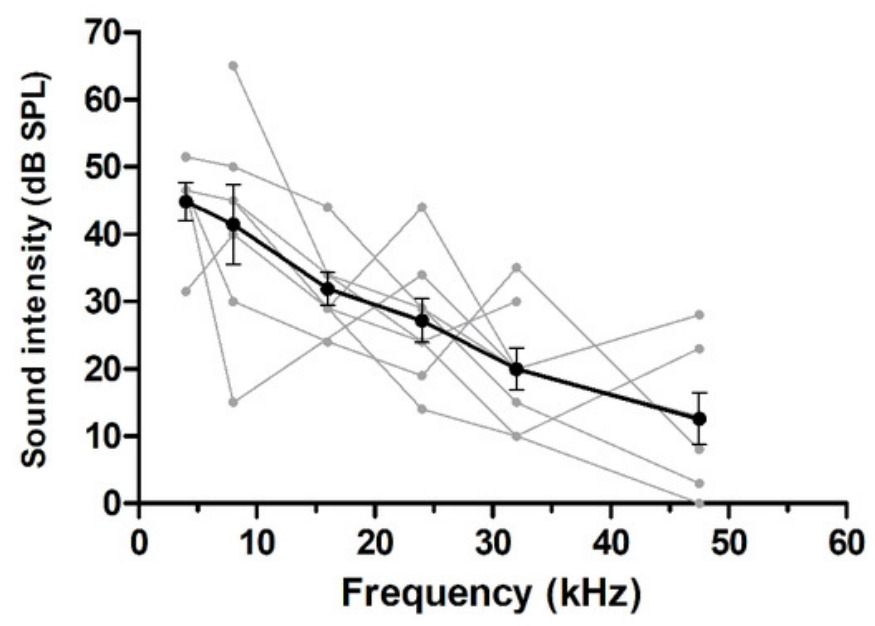

d

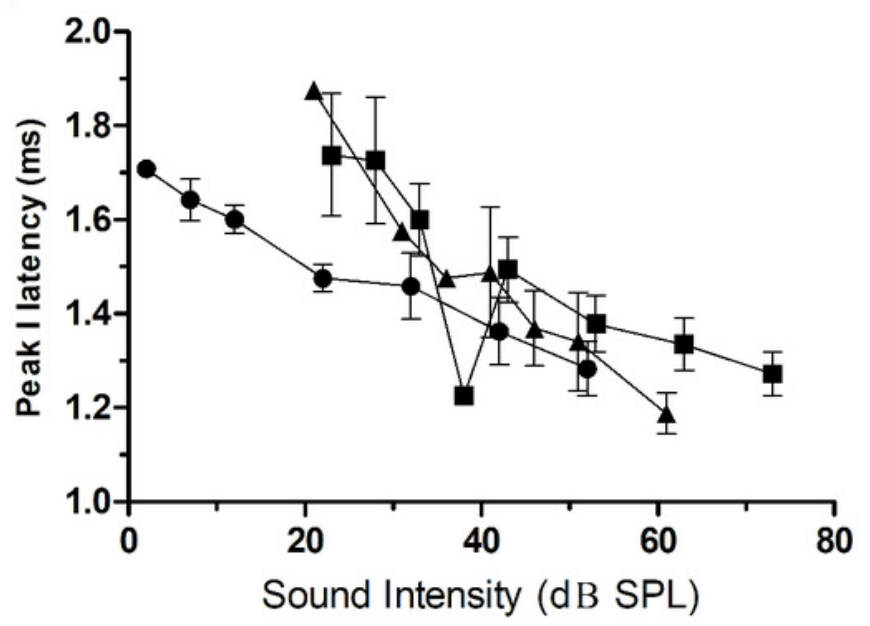




\section{Figure 3}

Overview of the fat-tailed dunnart auditory brainstem.

Nissl staining of transverse sections reveals prominent auditory nerve root nucleus and cochlear nuclei. Images are organised caudal to rostral. The dorsal cochlear nucleus resides medio-dorsal to the restiform body in the caudal regions (shown in a, with high power image in b). (c and d): More rostrally the ventral cochlear nucleus shows prominently as well. (d), (e), and (f): further rostral the trilaminar arrangement of the dorsal cochlear nucleus is clearly visible (f) as well as the cochlear nerve root nucleus ( $g$ ). At more rostral level ( $h$ ) the ventral cochlear nucleus shows a separation between posteroventral and anteroventral

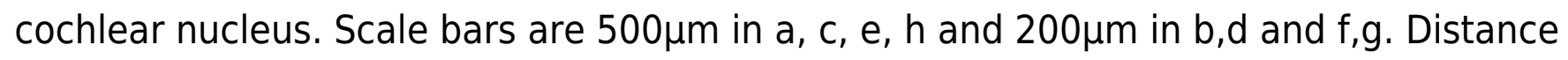

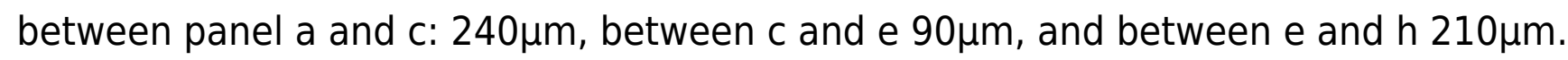
Section coordinates in panel ( $h$ ) are for all sections. Abbreviations: $\mathrm{cnr}$ - cochlear nerve root, $\mathrm{cb}$ - cerebellum, dcn - dorsal cochlear nucleus, fn - facial nucleus, rb - restiform body, avcn anteroventral cochlear nucleus, pvcn- posteroventral cochlear nucleus, in panel f: I molecular later II - fusiform layer III - polymorphic layer 

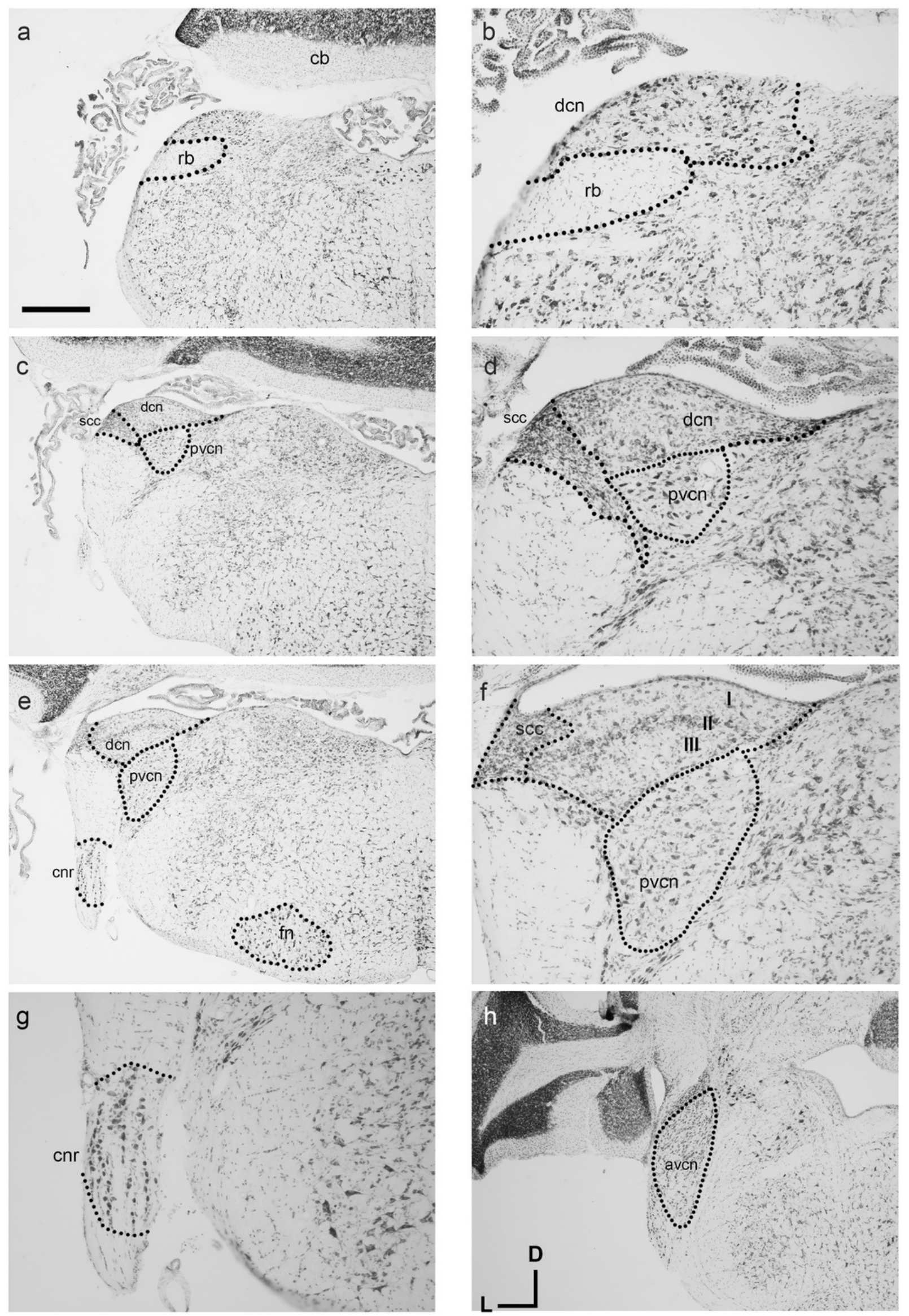


\section{Figure 4}

The superior olivary complex (SOC) nuclei in the fat-tailed dunnart.

The four main nuclei evident include the medial nucleus of the trapezoid body (MNTB) ( $a$ and

b) residing within the fibres of the trapezoid body (tb marked in a). The superior paraolivary nucleus (SPN) is located dorsomedial to the MNTB. Located laterally to the MNTB and SPN is the linear medial superior olive (MSO) ( $a, b$ with outline in $c$ ). The lateral superior olive (LSO) (outline in c) can be seen lateral to the MSO and contains a marginal (Iso-m) and core (Iso-c) regions (outlines in $\mathrm{a}, \mathrm{c}$ ). The boundary of the LSO shown in panel $\mathrm{c}$ is tentative and derive from alignment of neuronal somata. Micrographs are taken at $2 x(a)$ and 10x (b). Section coordinates in panel (c) are for all sections. Scale bars denote $1 \mathrm{~mm}$ in a and $200 \mathrm{~mm}$ in b, c. Abbreviations: Iso - lateral superior olive, mntb - medial nucleus of the trapezoid body, mso medial superior olive, spn- superior paraolivary nucleus, tb - trapezoid body. 

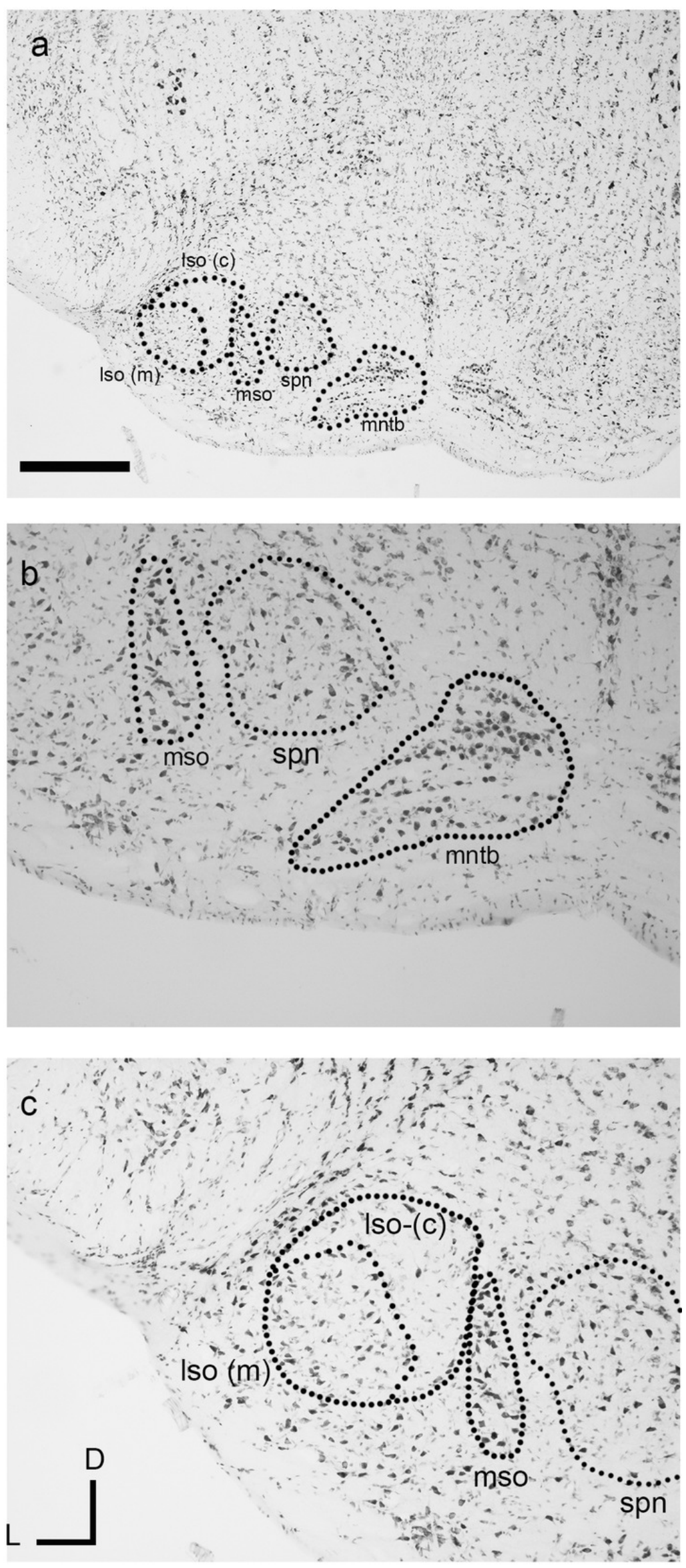

Peer) reviewing PDF | (2019:04:36946:1:1:NEW 22 Aug 2019) 